\title{
Ordering in weakly coupled random singlet spin chains
}

\author{
M. Thede, ${ }^{1,2}$ F. Xiao, ${ }^{1,3}$ Ch. Baines, ${ }^{2}$ C. Landee, ${ }^{3}$ E. Morenzoni, ${ }^{2}$ and A. Zheludev ${ }^{1, *}$ \\ ${ }^{1}$ Neutron Scattering and Magnetism, Laboratory for Solid State Physics, ETH Zürich, Zürich, Switzerland \\ ${ }^{2}$ Laboratory for Muon Spin Spectroscopy, Paul Scherrer Insitut, Villigen-PSI, Switzerland \\ ${ }^{3}$ Department of Physics, Clark University, 950 Main St., Worcester, Massachusetts 01610, USA
}

(Received 31 August 2012; published 29 November 2012)

\begin{abstract}
The influence of bond randomness on long-range magnetic ordering in the weakly coupled $S=1 / 2$ antiferromagnetic spin chain materials $\mathrm{Cu}(\mathrm{py})_{2}\left(\mathrm{Cl}_{1-x} \mathrm{Br}_{x}\right)_{2}$ is studied by muon spin rotation and bulk measurements. Disorder is found to have a strong effect on the ordering temperature $T_{\mathrm{N}}$, and an even stronger one on the saturation magnetization $m_{0}$, but considerably more so in the effectively lower-dimensional Br-rich materials. The observed behavior is attributed to random singlet ground states of individual spin chains, but remains in contradiction with chain mean-field theory [Joshi and Yang, Phys. Rev. B 67, 174403 (2003)] predictions. In this context, we discuss the possibility of a universal distribution of ordered moments in the weakly coupled random singlet chains model.
\end{abstract}

DOI: 10.1103/PhysRevB.86.180407

PACS number(s): 75.10.Pq, 75.10.Nr, 75.40.Cx, 75.50.Lk

The ground states of unfrustrated classical systems are typically robust with respect to weak Hamiltonian disorder. A case in point is the classical Heisenberg antiferromagnet (HAF). Randomizing the strength (but not the signs) of exchange interactions leaves the fully aligned Neel ground state completely intact. In contrast, in quantum systems, arbitrarily weak disorder will modulate the strengths of local quantum fluctuation and often qualitatively reconstruct the ground state. The one-dimensional quantum $S=1 / 2 \mathrm{HAF}$ is an extreme example. For uniform chains, the ground state is a Tomonaga-Luttinger spin liquid (TLSL). ${ }^{1}$ The introduction of arbitrary weak bond randomness gives rise to the so-called random singlet (RS) phase. ${ }^{2-5}$ In the RS state, spin correlations are protected from localization effects by particle-hole symmetry. ${ }^{1,6}$ Nevertheless, the scaling laws, ${ }^{5,7,8}$ although universal and independent of the details of disorder, are markedly different from those of the TLSL. In experiments on real materials, one has to deal with quasi-one-dimensional (quasi-1D) spin systems. A divergent correlation length in individual chains ensures three-dimensional (3D) long-range order at $T_{\mathrm{N}}>0$ for arbitrary weak interchain interactions $J^{\prime}$, in both the disorder-free ${ }^{9}$ and disordered cases. ${ }^{5,10}$ An intriguing question is to what extent the peculiarities of the RS state in $d=1$ translate into unusual features of the ordered phase in $d=3$.

To date, RS-forming bond randomness in quasi-1D magnets has received considerably less attention than dilution-type disorder due to spin substitution. ${ }^{11-13}$ For the former, the existing predictions are derived from chain-mean-field (chainMF) theory. ${ }^{10}$ The main result is that random bonds tend to increase both $T_{\mathrm{N}}$ and the ordered moment $m_{0}$ at $T \rightarrow 0$. This peculiar "order from disorder" effect is related to an abundance of very loosely coupled and almost free spins in the RS state of isolated chains. ${ }^{2,4}$ In origin, it is similar to disorder-induced ordering in frustrated magnets, where spin fluctuations are also strong. ${ }^{14,15}$ In coupled random chains, for weak $J^{\prime}$, one gets $T_{\mathrm{N}} \propto J^{\prime} m_{0} \cdot{ }^{10}$ In contrast, for the disorder-free case, the in-chain interactions enter the relation explicitly: $T_{\mathrm{N}} \propto J m_{0}^{2} \cdot{ }^{16}$ On the experimental side, the challenge is to measure the very small sublattice magnetization that arises in the weak-coupling regime, where this theory may be expected to apply. In the present work we overcome this difficulty by employing the sensitive muon spin rotation ( $\mu \mathrm{SR})$ technique, which has emerged as a tool of choice for the study of quantum magnetism. ${ }^{17}$ We directly measure the relative variations of $m_{0}$ and $T_{\mathrm{N}}$ in the prototypical bond-disordered quasi-1D $S=1 / 2$ HAF systems $\mathrm{Cu}(\mathrm{py})_{2}\left(\mathrm{Cl}_{1-x} \mathrm{Br}_{x}\right)_{2}$. The observed behavior, while starkly different from that in disorder-free chains, is in apparent contradiction with chain-MF predictions for coupled RS chains.

Our target compounds are derivatives of $\mathrm{Cu}(\mathrm{py})_{2} \mathrm{Cl}_{2}$, one of the first known and extensively studied $S=1 / 2$ spin chain materials. ${ }^{18}$ Single crystal samples with varying $\mathrm{Br}$ content $x$ are straightforward to grow from solution by slow evaporation. In $\mathrm{Cu}(\mathrm{py})_{2} \mathrm{Cl}_{2}$ (space group $P 2_{1} / n, a=16.967 \AA, b=$ $8.5596 \AA, c=3.8479 \AA, \beta=91.98^{\circ}$ ) the chains are formed by magnetic $S=1 / 2 \mathrm{Cu}^{2+}$ ions linked by superexchange bonds via the halogen sites. The temperature dependence of magnetic susceptibility [Fig. 1(a)] shows a broad Bonner-Fischer (BF) maximum, ${ }^{19,20}$ characteristic of a quantum $S=1 / 2$ chain with an antiferromagnetic exchange constant $J_{x=0}=2.35 \mathrm{meV}$. The chain structure of $\mathrm{Cu}(\mathrm{py})_{2} \mathrm{Br}_{2}$ is quite similar $\left[P 2_{1} / n, a=\right.$ $8.424 \AA, b=17.599 \AA, c=4.0504 \AA, \beta=97.12^{\circ}$ (Ref. 21 )], as are the measured magnetic susceptibility curves. However, the in-chain exchange constant is larger: $J_{x=1}=4.58 \mathrm{meV}$. Weak interchain interactions lead to $3 \mathrm{D}$ ordering in both materials, at $T_{\mathrm{N}}=1.15 \mathrm{~K}$ (Ref. 22) and $T_{\mathrm{N}}=0.72 \mathrm{~K}$ for $\mathrm{Cu}(\mathrm{py})_{2} \mathrm{Cl}_{2}$ and $\mathrm{Cu}(\mathrm{py})_{2} \mathrm{Br}_{2}$, respectively. The transitions are marked by well-defined $\lambda$ anomalies in the measured temperature dependence of specific heat $C(T)$, as shown in Fig. 1(b). ${ }^{23}$ Knowing $T_{\mathrm{N}}$ and $J$, allows us to estimate the effective interchain coupling constants: ${ }^{24} J_{x=0}^{\prime}=0.05 \mathrm{meV}$ and $J_{x=1}^{\prime}=0.03 \mathrm{meV}$ for the two materials, correspondingly. The bromide is clearly a much more one-dimensional system. It is useful to estimate the ordered moment at $T \rightarrow 0$. Based on chain-MF results, for $\mathrm{Cu}(\mathrm{py})_{2} \mathrm{Cl}_{2}$ and $\mathrm{Cu}(\mathrm{py})_{2} \mathrm{Br}_{2}$ we get $m_{0, x=0}=0.15 \mu_{\mathrm{B}}$ and $m_{0, x=1}=0.08 \mu_{\mathrm{B}}$, respectively. The smaller ordered moment, and hence a greater one-dimensional character of the $\mathrm{Br}$ system, is also manifested in the much weaker $C(T) \lambda$ anomaly. 

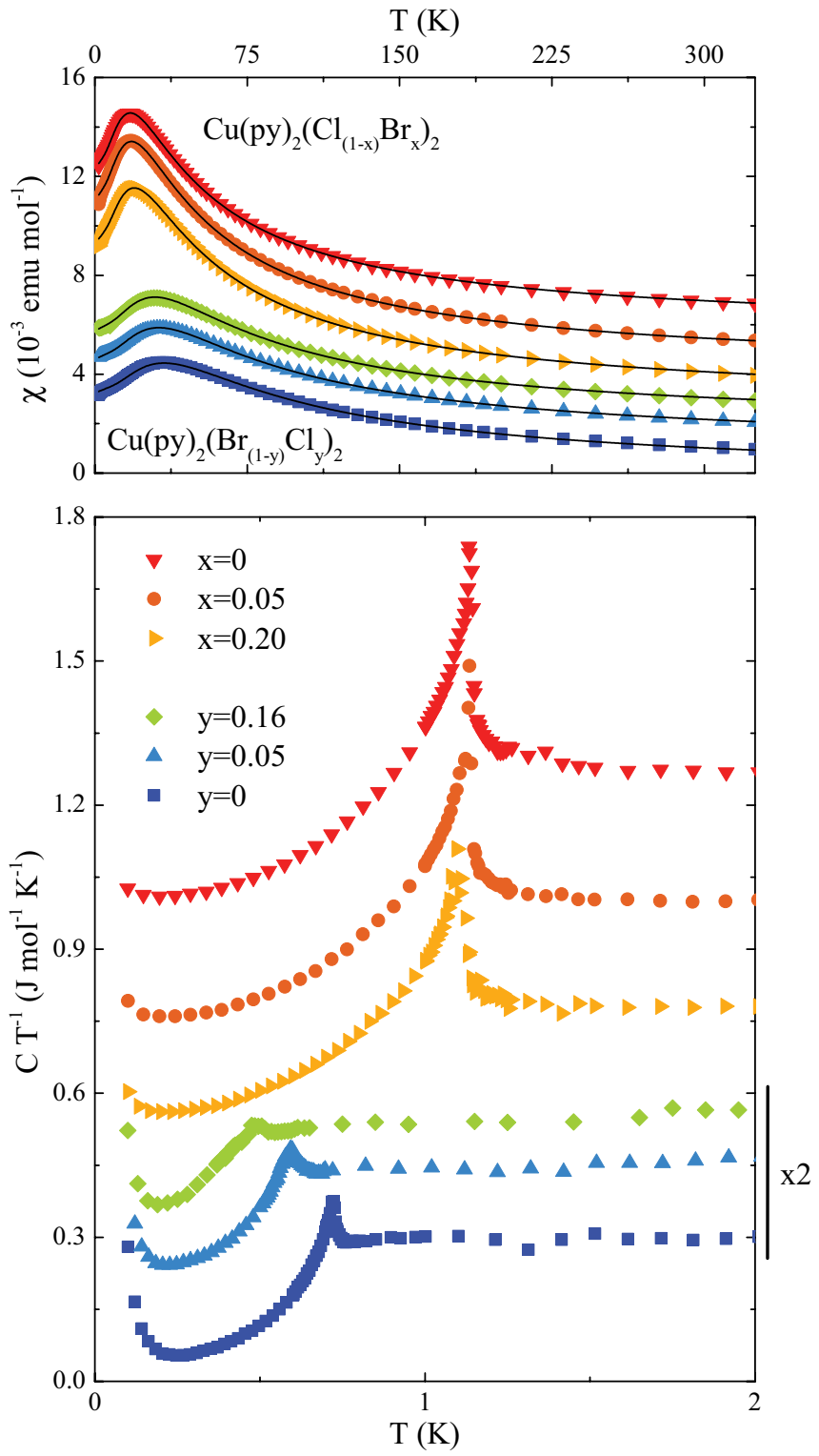

FIG. 1. (Color online) Bulk properties of $\mathrm{Cu}(\mathrm{py})_{2}\left(\mathrm{Cl}_{1-x} \mathrm{Br}_{x}\right)_{2}$ samples. (a) Measured temperature dependence of magnetic susceptibility for a field applied along the chain axis (symbols), and fit of the theoretical curve for the uniform quantum $S=1 / 2$ HAF chain (Refs. 19 and 20) (solid line). From the bottom up, the data are offset along the $y$ axis by $0,1,2,3,4.2$, and $5.8 \times 10^{-3} \mathrm{emu} / \mathrm{mol}$, respectively. (b) Measured temperature dependence of specific heat (symbols). The vertical offsets are $0,0.16,0.27,0.55,0.75$, and $1 \mathrm{~J} / \mathrm{mol} \mathrm{K}$, respectively.

As determined by single crystal x-ray diffraction and chemical elemental analysis, the structures of $\mathrm{Cu}(\mathrm{py})_{2} \mathrm{Cl}_{2}$ and $\mathrm{Cu}(\mathrm{py})_{2} \mathrm{Br}_{2}$ are stable with respect to chemical substitution of $\mathrm{Cl}$ for $\mathrm{Br}$ and vice versa, for $x<0.4$ and $x>0.6$, respectively. In addition to changing bond angles due to a difference in ionic radii, the more extended wave functions in $\mathrm{Br}^{-}$provide a stronger superexchange pathway compared to $\mathrm{Cl}^{-}$, typically by a factor of 2 to $4 .{ }^{25}$ This strategy of creating bond-disordered systems has previously been successfully applied in other materials such as IPA-Cu( $\left.\mathrm{Cl}_{1-x} \mathrm{Br}_{x}\right)_{3},{ }^{26}$ piperazinium$\mathrm{Cu}_{2}\left(\mathrm{Cl}_{1-x} \mathrm{Br}_{x}\right)_{6},{ }^{27} \quad \mathrm{H}_{8} \mathrm{C}_{4} \mathrm{SO}_{2} \cdot \mathrm{Cu}_{2}\left(\mathrm{Cl}_{1-x} \mathrm{Br}_{x}\right)_{4},{ }^{28}$ and

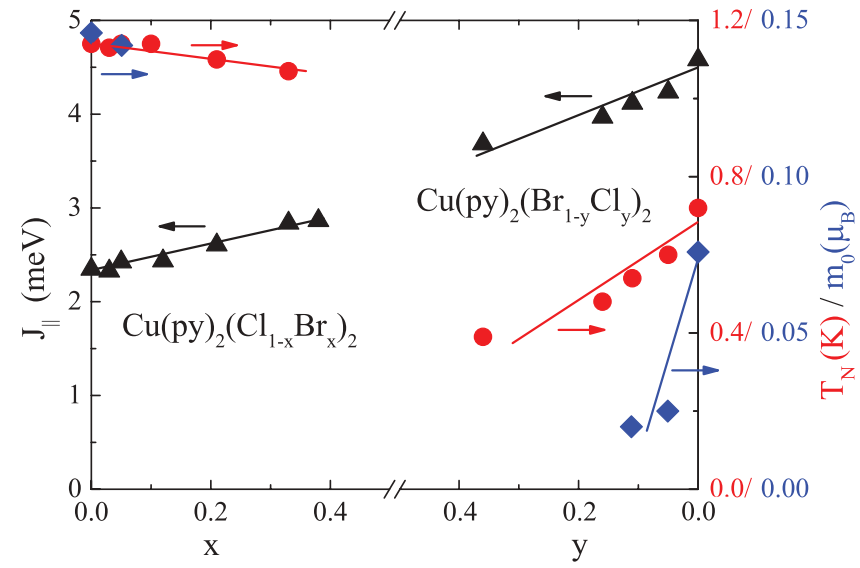

FIG. 2. (Color online) Composition dependence of the in-chain exchange constant $J$ [from fits to $\chi(T)$ ], the ordering temperature $T_{\mathrm{N}}$ (from calorimetry), and the low-temperature ordered moment $m_{0}$ (chain-MF estimates and $\mu$-SR measurements).

$\mathrm{NiCl}_{2} \cdot 4 \mathrm{SC}\left(\mathrm{NH}_{2}\right)_{2} \cdot{ }^{29}$ Due to the slightly different structures of the parent compounds, we are actually dealing with two tunable random-bond spin chain materials, on the $\mathrm{Cl}$-rich and $\mathrm{Br}$-rich ends of the $\mathrm{Cu}(\mathrm{py})_{2}\left(\mathrm{Cl}_{1-x} \mathrm{Br}_{x}\right)_{2}$ line, respectively. We will reserve the formula $\mathrm{Cu}(\mathrm{py})_{2}\left(\mathrm{Cl}_{1-x} \mathrm{Br}_{x}\right)_{2}$ for Cl-rich compounds $(x<0.5)$ and use $\mathrm{Cu}(\mathrm{py})_{2}\left(\mathrm{Br}_{1-y} \mathrm{Cl}_{y}\right)_{2}$ to denote materials on the $\mathrm{Br}$ end $(y<0.5)$.

The bulk properties of the halogen-disordered samples resemble those of the corresponding disorder-free systems. Typical measured magnetic susceptibility data are plotted symbols in Fig. 1. The derived average in-chain exchange constant $J$ steadily increases with increasing $\mathrm{Br}$ content. A 3D magnetic ordering transition is observed at low temperatures in all compositions studied (Fig. 1, lower panel). $T_{\mathrm{N}}$ decreases with $\mathrm{Br}$ content on the $\mathrm{Cl}$-rich end and with $\mathrm{Cl}$ concentration in $\mathrm{Br}$-rich samples (Fig. 2). The variation is more pronounced in $\mathrm{Cu}(\mathrm{py})_{2}\left(\mathrm{Br}_{1-y} \mathrm{Cl}_{y}\right)_{2}$. Moreover, the corresponding $\lambda$ anomaly weakens and slightly broadens with increasing $\mathrm{Cl}$ content in $\mathrm{Cu}(\mathrm{py})_{2}\left(\mathrm{Br}_{1-y} \mathrm{Cl}_{y}\right)_{2}$, while it remains almost unchanged in $\mathrm{Cu}(\mathrm{py})_{2}\left(\mathrm{Cl}_{1-x} \mathrm{Br}_{x}\right)_{2}$. The measured transition temperature and average exchange constant are plotted versus composition in Fig. 2.

The main focus of the present study is on $\mu$-SR measurements. This technique has been instrumental in the study of very small static $c^{30,31}$ and dynamic ${ }^{32,33}$ moments in spin chain systems. It probes the local magnetic fields at the stopping sites of muons implanted into the sample. ${ }^{34}$ These, in turn, are expected to be proportional to the static ordered moment. We performed measurements on powder samples of $\mathrm{Cu}(\mathrm{py})_{2}\left(\mathrm{Cl}_{1-x} \mathrm{Br}_{x}\right)_{2}$ and $\mathrm{Cu}(\mathrm{py})_{2}\left(\mathrm{Br}_{1-y} \mathrm{Cl}_{y}\right)_{2}$ with $x=0,0.05$ and $y=0,0.05$ and 0.1 at the LTF spectrometer at the $\mathrm{S} \mu \mathrm{S}$ muon source at Paul Scherrer Institut. Typical muon spin relaxation/rotation curves measured in zero applied field (ZF) are shown in Fig. 3. Several distinct time scales are apparent. In all samples, a very rapid decay at short times can be attributed to muonium formation with the organic ligand. ${ }^{35}$ This contribution appears to be temperature independent and is not directly relevant to the physics discussed here. At the largest time scales, a slowly decaying tail is due to the 


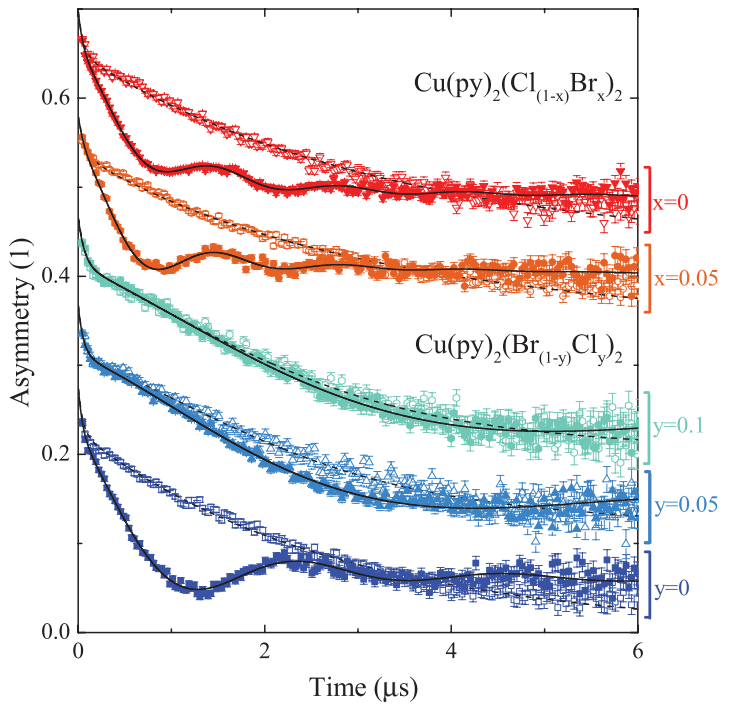

FIG. 3. (Color online) Muon spin asymmetry measured vs time in several $\mathrm{Cu}(\mathrm{py})_{2}\left(\mathrm{Cl}_{1-x} \mathrm{Br}_{x}\right)_{2}$ and $\mathrm{Cu}(\mathrm{py})_{2}\left(\mathrm{Br}_{1-y} \mathrm{Cl}_{y}\right)_{2}$ samples at $T \sim 20 \mathrm{mK}$ (solid symbols) and just above $T_{\mathrm{N}}$ (open symbols). The plots for $y=0,0.05$, and 0.1 and $x=0.05,0$ are offset along the $y$ axis by $0,0.1,0.2,0.3$, and 0.43 , respectively. The solid lines are fits to the data as described in the text.

nonprecessing muon spin components parallel to the local field, and to muons stopping outside the sample. ${ }^{34}$

As expected, in the paramagnetic phases of all samples one only observes a slow decay of muon polarization, with no oscillatory behavior. Representative data collected at $T>T_{\mathrm{N}}$ are shown by open symbols in Fig. 3. They can be modeled with exponential decay processes, as shown by dashed lines. We conclude that above the ordering transition, all materials studied behave very similarly, despite the different levels of disorder.

Clear differences emerge at low temperatures, in the magnetically ordered phase (Fig. 3, solid symbols). Although the relevant time scales turned out to be strongly dependent on composition, it is possible to provide a common description of the low-temperature $\mu$-SR spectra in all samples. In addition to the fast and slow background contributions described above, for $T<T_{\mathrm{N}}$, the main effect is the appearance of spontaneous muon spin precession. An application of a small longitudinal field parallel to the muon spin direction recovered all polarization apart from the muonium contribution. This observation shows that the observed spin relaxation and rotation are due to static internal fields, which we attribute to ordered $\mathrm{Cu}^{2+}$ moments. We chose to model these processes as a sum of a damped Bessel function and an exponential term. The spectra are then described as

$$
\begin{aligned}
A(t)= & A_{1} J_{0}(\omega t+\phi) \exp \left(-\lambda_{1} t\right)+A_{2} \exp \left(-\lambda_{2} t\right) \\
& +A_{\text {fast }} \exp \left(-\lambda_{\text {fast }} t\right)+A_{\text {tail }} \exp \left(-\lambda_{\text {tail }} t\right) .
\end{aligned}
$$

The first term on the right-hand side represents the precession of muons stopped at the most probable sites. ${ }^{32}$ The choice of a Bessel function, typically used to describe incommensurate structures, ${ }^{34}$ is in our case purely empirical. Nevertheless, its use is justified by preliminary neutron diffraction evidence ${ }^{36}$ that the magnetic structure is actually

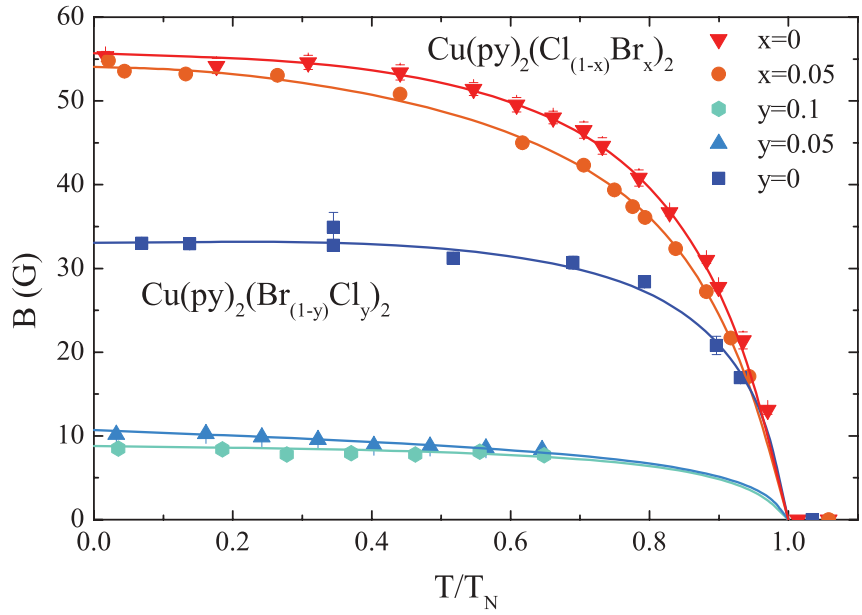

FIG. 4. (Color online) Characteristic field at the principal muon stopping site, assumed to be proportional to the magnetic order parameter, plotted against temperature in units of the average in-chain exchange constant $J$ in $\mathrm{Cu}(\mathrm{py})_{2}\left(\mathrm{Cl}_{1-x} \mathrm{Br}_{x}\right)_{2}$ and $\mathrm{Cu}(\mathrm{py})_{2}\left(\mathrm{Br}_{1-y} \mathrm{Cl}_{y}\right)_{2}$ (symbols). Within each family of materials, the relative magnitudes of the precession field can be directly compared. Lines are guides to the eye.

helimagnetic in the $b$ direction, perpendicular to the chains. Its exponential envelope reflects a narrow Lorentzian distribution of local fields and also takes into account the depolarization by nuclear spins. The characteristic muon spin precession frequency $\omega$ is directly proportional to the magnitude of the static magnetic field $\omega=\gamma B$, with $\gamma=85.16 \mathrm{krad} \mathrm{s}^{-1} \mathrm{G}^{-1}$, is thus our primary measure of the static magnetic order.

The second term in Eq. (1) empirically describes the multitude of other stopping sites sensitive to the static magnetic order. For each sample, the site occupancies $A_{1}$ and $A_{2}$, as well as parameters for "fast" and "tail" contributions, were determined in global fits to the data collected in the entire temperature range. The parameter $\phi$ determines the functional shape of the oscillatory term. At each temperature, it was globally applied to all samples, separately on the Br-rich and Cl-rich ends, to allow a direct comparison of the precession frequencies $\omega$ within each family of materials. For each sample, the parameters $\omega, \lambda_{1}$, and $\lambda_{2}$ were refined at each temperature separately. The model provides excellent fits to all data collected for the ordered state in all samples. Typical fits are shown in heavy solid lines in Fig. 3. The values of all fit parameters for all samples and their temperature dependencies are deposited as Supplemental Material. ${ }^{37}$

The main result of our analysis is the temperature dependence of the local field $B$, plotted in Fig. 4. For each composition, the temperature axis has been normalized to the value of $T_{c}$ derived from calorimetric measurements. Three conclusions can be immediately drawn. First, in apparent contradiction with chain-MF theory, both $T_{\mathrm{N}}$ and the saturation magnetization $m_{0}$ are reduced in disordered samples, on both composition ends. Second, the Br-rich materials are considerably more affected by disorder than the Cl-rich systems. Here, the oscillatory term is dramatically slowed already at 5\% substitution, and is almost completely overrun by relaxation effects at $10 \% \mathrm{Cl}$. Experimental conditions and data quality are very similar in all five samples, so the disappearance 
of clear oscillations is direct proof of $m_{0}$ reduction. This effect is fully consistent with a progressive weakening of the $C(T)$ $\lambda$ anomaly in $\mathrm{Cu}(\mathrm{py})_{2}\left(\mathrm{Br}_{1-y} \mathrm{Cl}_{y}\right)_{2}$ with increasing $y$ (Fig. 1). Third, for Br-rich samples the effect of bond disorder on $m_{0}$ is much more drastic than on $T_{\mathrm{N}}$. This is made particularly clear by the $m_{0}$ vs composition plot in Fig. 2. It is based on our initial estimates for $m_{0}$ in the disorder-free materials, and on the assumption that within each of the two series of materials $m_{0}$ is proportional to the Larmor field $B$ extrapolated to zero temperature.

That disorder effects are not apparent in bulk properties above $T_{\mathrm{N}}$ is not at all surprising. Recent numerical simulations and experimental studies of the RS material $\mathrm{BaCu}_{2} \mathrm{SiGeO}_{7}$ (Ref. 38) have illustrated that the bulk effect of even very large randomness may be very modest. Deviant behavior of susceptibility (in our case measured down to $2 \mathrm{~K}$ ) may emerge only at temperatures that are an order of magnitude smaller than the width of the bond probability distribution $P(J)$. Even if we assume that in $\mathrm{Cu}(\mathrm{py})_{2}\left(\mathrm{Br}_{1-y} \mathrm{Cl}_{y}\right)_{2}$ we are dealing with a clearly exaggerated bimodal distribution, a $10 \%$ substitution will produce a standard deviation of only $\sim 0.7 \mathrm{meV}$. In this conservative estimate, RS behavior may be expected to affect the bulk properties only at temperatures below $\sim 1 \mathrm{~K}$. As far as the specific heat is concerned, for RS chains one expects a power-law behavior with $C(T) \propto T^{\gamma_{\mathrm{C}}}{ }^{2}$ The exponents depend on the actual $P(J)$, and show a slow temperature dependence. For the relevant temperature range in our experiments $\left(T_{\mathrm{N}}<\right.$ $T \lesssim 5 \mathrm{~K}$ ), for $P(J)$ with a support removed from $J=0$ (most certainly true in our case), Ref. 2 suggests $0.9<\gamma_{\mathrm{C}}<1.1$. This explains why, despite the disorder, $C(T) / T$ remains roughly constant above $T_{\mathrm{N}}$ in all of our samples.

A key point is that for three-dimensional ordering in at least for the Br-rich materials, disorder has to be relevant. Specifically, for $y=0.05$, from the measured values of $J$ and $T_{\mathrm{N}}$, from chain-MF theory for disorder-free chains, ${ }^{16}$ one gets $m_{0} \approx 0.06 \mu_{\mathrm{B}}$, as compared to the much smaller observed value $m_{0} \approx 0.025 \mu_{\mathrm{B}}$. The discrepancy cannot be explained without invoking disorder effects, and is even more drastic for $y=0.1: m_{0} \approx 0.06 \mu_{\mathrm{B}}$ or a disorder-free model vs $m_{0}<$
$0.02 \mu_{\mathrm{B}}$ observed. In contrast, the mismatch between $T_{\mathrm{N}}$ and $m_{0}$ in $\mathrm{Cu}(\mathrm{py})_{2}\left(\mathrm{Cl}_{1-x} \mathrm{Br}_{x}\right)_{2}$, as compared to expectations for disorder-free chains, is not as drastic. To explain this, we recall that RS properties emerge only below a certain energy scale that is nonuniversal and depends on the initial distribution of exchange constants. ${ }^{2-5} \mathrm{In} \mathrm{Cu}(\mathrm{py})_{2}\left(\mathrm{Cl}_{1-x} \mathrm{Br}_{x}\right)_{2}$, where the relative strength of interchain interaction is roughly four times stronger than in $\mathrm{Cu}(\mathrm{py})_{2}\left(\mathrm{Br}_{1-y} \mathrm{Cl}_{y}\right)_{2}$, this energy scale may be much lower than $3 \mathrm{D}$ interactions, making disorder irrelevant in the ordered phase.

The discrepancy between our findings and the chain-MF theory of Ref. 10 remains to be explained. One tantalizing possibility is that the chain-MF approach may, in principle, be inapplicable to the RS phase. ${ }^{39}$ The latter features an abundance of weakly dimerized spin degrees of freedom for which quantum correlations with similar objects in adjacent chains simply cannot be ignored. A final note concerns the homogeneity of the static ordered moment. The low-energy physics of the RS phase is exactly that of noninteracting random dimers with a universal probability distribution of dimer strength. These singlets will be partially polarized by the mean exchange field. The degree of polarization will be determined by the strength of the dimers. As a result, we expect a universal probability and spatial distribution of static ordered moments $P(m)$ in the $T \rightarrow 0$ limit. Even though muon spectroscopy could, in principle, measure this distribution directly, in $\mathrm{Cu}(\mathrm{py})_{2}\left(\mathrm{Cl}_{1-x} \mathrm{Br}_{x}\right)_{2}$ and $\mathrm{Cu}(\mathrm{py})_{2}\left(\mathrm{Br}_{1-y} \mathrm{Cl}_{y}\right)_{2}$ such an experiment appears very challenging. We believe that this elegant idea of a universal distribution of ordered moments in weakly coupled RS chains deserves more theoretical and experimental attention, using new materials for $\mu$-SR and using alternative techniques such as NMR.

This work is partially supported by the Swiss National Fund under project 2-77060-11 and through Collaborative Project 8 of MANEP. We would like to thank Mark Turnbull for helpful discussions of the single crystal growth and diffraction analysis, and Hubertus Luetkens for help and guidance in analyzing the muon data. *zhelud@ethz.ch; http://www.neutron.ethz.ch/

${ }^{1}$ T. Giamarchi, Quantum Physics in One Dimension (Clarendon, Oxford, 2003).

${ }^{2}$ S.-k. Ma, C. Dasgupta, and C.-k. Hu, Phys. Rev. Lett. 43, 1434 (1979).

${ }^{3}$ C. Dasgupta and S.-k. Ma, Phys. Rev. B 22, 1305 (1980).

${ }^{4}$ C. A. Doty and D. S. Fisher, Phys. Rev. B 45, 2167 (1992).

${ }^{5}$ D. S. Fisher, Phys. Rev. B 50, 3799 (1994).

${ }^{6}$ G. Theodorou and M. H. Cohen, Phys. Rev. B 13, 4597 (1976)

${ }^{7}$ K. Damle, O. Motrunich, and D. A. Huse, Phys. Rev. Lett. 84, 3434 (2000).

${ }^{8}$ O. Motrunich, K. Damle, and D. A. Huse, Phys. Rev. B 63, 134424 (2001).

${ }^{9}$ D. J. Scalapino, Y. Imry, and P. Pincus, Phys. Rev. B 11, 2042 (1975).

${ }^{10}$ A. Joshi and K. Yang, Phys. Rev. B 67, 174403 (2003).
${ }^{11}$ R. J. Birgeneau, R. A. Cowley, G. Shirane, J. A. Tarvin, and H. J. Guggenheim, Phys. Rev. B 21, 317 (1980).

${ }^{12}$ Y. Endoh, I. U. Heilmann, R. J. Birgeneau, G. Shirane, A. R. McGurn, and M. F. Thorpe, Phys. Rev. B 23, 4582 (1981).

${ }^{13}$ Y. Uchiyama, Y. Sasago, I. Tsukada, K. Uchinokura, A. Zheludev, T. Hayashi, N. Miura, and P. Böni, Phys. Rev. Lett. 83, 632 (1999). ${ }^{14}$ C. L. Henley, Phys. Rev. Lett. 62, 2056 (1989).

${ }^{15}$ A. Chubukov, Phys. Rev. Lett. 69, 832 (1992).

${ }^{16}$ H. J. Schulz, Phys. Rev. Lett. 77, 2790 (1996).

${ }^{17}$ F. L. Pratt, P. J. Baker, S. J. Blundell, T. Lancaster, S. OhiraKawamura, C. Baines, Y. Shimizu, K. Kanoda, I. Watanabe, and G. Saito, Nature (London) 471, 612 (2011).

${ }^{18}$ Y. Endoh, G. Shirane, R. J. Birgeneau, P. M. Richards, and S. L. Holt, Phys. Rev. Lett. 32, 170 (1974).

${ }^{19}$ J. C. Bonner and M. E. Fisher, Phys. Rev. 135, A640 (1964). 
${ }^{20}$ D. C. Johnston, R. K. Kremer, M. Troyer, X. Wang, A. Klumper, S. L. Budko, A. F. Panchula, and P. C. Canfield, Phys. Rev. B 61, 9558 (2000).

${ }^{21}$ B. Morosin, Acta Crystallogr. B 31, 632 (1975).

${ }^{22}$ K. Takeda, Y. Yamamoto, and T. Haseda, Phys. Lett. 45, 419 (1973).

${ }^{23}$ All specific heat data shown in this Rapid Communication were measured on single crystals using a Quantum Design Physical Properties Measurement system PPMS-XL with a ${ }^{3} \mathrm{He}-{ }^{4} \mathrm{He}$ dilution refrigerator insert.

${ }^{24}$ C. Yasuda, S. Todo, K. Hukushima, F. Alet, M. Keller, M. Troyer, and H. Takayama, Phys. Rev. Lett. 94, 217201 (2005).

${ }^{25}$ R. D. Willett, Inorg. Chem. 25, 1918 (1986).

${ }^{26}$ T. Hong, A. Zheludev, H. Manaka, and L.-P. Regnault, Phys. Rev. B 81, 060410 (2010).

${ }^{27}$ D. Hüvonen, S. Zhao, M. Mansson, T. Yankova, E. Ressouche, C. Niedermayer, M. Laver, S. N. Gvasaliya, and A. Zheludev, Phys. Rev. B 85, 100410 (2012).

${ }^{28}$ E. Wulf, S. Mühlbauer, T. Yankova, and A. Zheludev, Phys. Rev. B 84, 174414 (2011).

${ }^{29}$ R. Yu et al., Nature 489, 379 (2012).
${ }^{30}$ T. Lancaster, S. J. Blundell, M. L. Brooks, P. J. Baker, F. L. Pratt, J. L. Manson, C. P. Landee, and C. Baines, Phys. Rev. B 73, 020410 (2006).

${ }^{31}$ T. Sugano, S. J. Blundell, T. Lancaster, F. L. Pratt, and H. Mori, Phys. Rev. B 82, 180401 (2010).

${ }^{32}$ J. A. Chakhalian, R. F. Kiefl, R. Miller, J. Brewer, S. R. Dunsiger, G. Morris, W. A. MacFarlane, J. E. Sonier, S. Eggert, I. Affleck, A. Keren, and M. Verdaguer, Phys. Rev. Lett. 91, 027202 (2003).

${ }^{33}$ F. L. Pratt, S. J. Blundell, T. Lancaster, C. Baines, and S. Takagi, Phys. Rev. Lett. 96, 247203 (2006).

${ }^{34}$ A. Yaouanc and P. D. Reotier, Muon Spin Rotation, Relaxation and Resonance (Oxford University Press, New York, 2011).

${ }^{35}$ C. G. Rhodes, H. Morris, and I. D. Reid, Magn. Reson. Chem. 39, 438 (2001).

${ }^{36} \mathrm{M}$. Thede (unpublished).

${ }^{37}$ See Supplemental Material at http://link.aps.org/supplemental/ 10.1103/PhysRevB.86.180407 for a complete summary of all parameter values obtained in the least squares analysis of the $\mu$-SR data.

${ }^{38}$ T. Shiroka, F. Casola, V. Glazkov, A. Zheludev, K. Prsa, H. R. Ott, and J. Mesot, Phys. Rev. Lett. 106, 137202 (2011).

${ }^{39} \mathrm{~T}$. Giamarchi (private communication). 